\title{
Estudio exploratorio de bacterias solubilizadoras de fósforo en dos hábitats asociados con Cedrela montana Moritz ex Turcz
}

\author{
An exploratory study of phosphorus-solubilizing bacteria in two habitats associated with \\ Cedrela montana Moritz ex Turcz
}

\section{Tanya Ascencio-Lino ${ }^{1 *}$, Esther Matamoros-Alcívar¹, Verónica Sandoya-Sánchez ${ }^{1}$, Milton Barcos-Arias², Jaime Naranjo-Morán²}

DOl. 10.21931/RB/2021.06.01.20

Resumen: La disponibilidad de fósforo $(P)$ en la corteza terrestre está limitada por la composición química del suelo, y por factores antropogénicos en el caso de ambientes perturbados. Debido a que solo el $0.1 \%$ de P está disponible para ser absorbido directamente por las plantas, se requiere de una interacción positiva con bacterias solubilizadoras de fósforo (BSF) para convertir el $\mathrm{P}$ insoluble a su forma soluble, y hacerlo disponible para las plantas. Aun siendo esta asociación de gran importancia, existe poca información sobre la presencia de BSF en especies arbóreas, especialmente de ambientes montanos andinos. El objetivo de esta investigación fue identificar la presencia de BSF en la rizosfera de dos individuos de cedro andino (Cedrela montana), provenientes de bosque secundario (BS) y zona de pastizal (ZP), respectivamente, de la localidad de Intag (norte de la Cordillera Occidental del Ecuador). Se recolectaron un total de tres muestras de suelo de cada rizósfera, las cuales fueron enriquecidas con fosfato tricálcico, y cultivadas en medio selectivo NBRIP para determinar la presencia de BSF. Se obtuvieron 54 aislados de BS y 46 de ZP, con capacidad de solubilización de fósforo. Los aislados BS-5, BS-6 y ZP-10 alcanzaron los índices de solubilización de fosfato más altos, con valores de 4.63, 2.75 y 2.08, respectivamente. Los resultados sugieren que las BSF, procedentes de suelo rizosférico de C. montana, están presentes en ambos hábitats (BS y ZP), donde fueron encontrados aislados promisorios; sin embargo, aquellos provenientes de BS mostraron una mayor capacidad solubilizadora de fósforo inorgánico. La información obtenida en este estudio servirá de base para el desarrollo futuro de bioinóculos específicos que permitan la propagación del cedro andino en condiciones de invernadero.

Palabras clave: Aislados promisorios, bacterias solubilizadoras de fósforo, bosque montano, bosque secundario, zona de pastizal, Cedrela montana.

\begin{abstract}
The availability of phosphorus (P) in the earth's crust is limited by the chemical composition of the soil, and by anthropogenic factors in disturbed environments. Only $0.1 \%$ of $P$ is available to be absorbed directly by plants, positive interaction with phosphorus solubilizing bacteria (PSB) is required to convert $\mathrm{P}$ insoluble to its soluble form and make it available to plants. Although this association is of great importance, there is little information over presence on BSF in tree species from Andean montane forests. The objective of this research was to identify the presence of BSF in the rhizosphere of two individuals of Andean cedar (Cedrela montana), from the secondary forest (BS) and grassland zone (ZP), located in Intag (north of the Western Cordillera of Ecuador). A total of three samples were collected from the soil of each rhizosphere, which was enriched with tricalcium phosphate, and cultured in NBRIP selective medium to determinate the presence of BSF. We obtained 54 BS isolates and 46 ZP isolates, with phosphorus solubilization capacity. The BS-5, BS-6, and ZP-10 reached the highest phosphate solubilization indices, with values of $4.63,2.75$, and 2.08 . The results suggest that BSF, from rhizospheric soil of $C$. montana, is present in both habitats (BS and ZP), where we found promising isolates; however, those from BS showed a greater solubilizing capacity of inorganic phosphorus. The information obtained in this study will serve as the basis for the future development of specific bioinoculants that allow the propagation of Andean cedar under greenhouse conditions.
\end{abstract}

Key words: Isolates, phosphorus solubilizing bacteria, montane forest, secondary forest, grassland zone, Cedrela montana.

\section{Introducción}

El fósforo $(P)$ es un nutriente esencial para las plantas, ya que es parte integral de sus actividades metabólicas y de desarrollo ${ }^{1,2}$. El P tiene un ciclo sedimentario y su presencia es limitada en el suelo; al no estar disponible en la atmósfera, los microorganismos son esenciales para mantener su ciclo natural. Este importante elemento químico es absorbido por las plantas a través de procesos de solubilización y mineralización ${ }^{1,3}$, en los cuales intervienen los microorganismos. Estos actúan como mediadores de la disponibilidad de $\mathrm{P}$, al liberarlo desde las reservas inorgánicas y orgánicas del suelo para aprovechamiento de las plantas. La solubilización del P media-

da por microorganismos es un proceso relevante y complejo, que depende de las condiciones nutricionales y fisiológicas de cada individuo ${ }^{3}$. Los principales microorganismos asociados a esta importante actividad son las bacterias solubilizadoras de fósforo (BSF).

Las BSF representan alrededor del 10\% de la población microbiana del suelo․ En especies arbóreas de lotes experimentales se han encontrado varias cepas de BSF del género Bacillus y Pseudomona 4 . Sin embargo, existe un vacío de información referente a BSF asociadas a especies forestales en bosques de los Andes tropicales.

\footnotetext{
${ }^{1}$ Universidad Yachay Tech, Escuela de Ciencias Biológicas e Ingeniería, Imbabura, Ecuador.

${ }^{2}$ Escuela Superior Politécnica del Litoral, ESPOL, Facultad de Ciencias de la Vida, FCV, Centro de Investigaciones Biotecnológicas del Ecuador, CIBE, Guayaquil, Ecuador.
} 
Nuestro estudio evalúa, la presencia de BSF en la rizósfera de una especie forestal, C. montana (cedro andino), distribuida en bosques montanos de la Cordillera Occidental de Ecuador $^{5}$. Esta especie se encuentra incluida en el apéndice II de la Convención sobre el Comercio Internacional de Especies Amenazadas de Fauna y Flora silvestres $\left(\right.$ CITES) ${ }^{6}$. Esta situación de amenaza se debe principalmente a su extracción indiscriminada desde áreas de distribución natural, debido a las cualidades de su madera ${ }^{7}$. Por otro lado, áreas con presencia de esta especie han estado expuestas a deforestación y conversión extensiva de bosques a pastizales, lo que ha ocasionado pérdida de fertilidad, calidad y biodiversidad del suelo ${ }^{8,9}$ Por tales razones, es indispensable obtener bacterias nativas solubilizadoras de P para incrementar a futuro la adaptación y crecimiento de $C$. montana en suelos con distinta cobertura vegetal.

\section{Materiales y métodos}

\section{Área de estudio}

Las muestras de suelo rizosférico de C. montana fueron colectadas en dos diferentes hábitats, BS y ZP, en la localidad de La Neblina - Intag, al norte de la Cordillera Occidental del Ecuador, en las coordenadas N $0^{\circ} 20^{\prime} 06.8^{\prime \prime}$ y W 78²5'33.5" (Figura 1). En cada hábitat se recolectaron, con un barreno y hasta una profundidad de $20 \mathrm{~cm}^{1}$, un total de tres muestras simples de suelo. Las muestras fueron homogeneizadas in situ, obteniendo así una muestra de suelo compuesta, de aproximadamente 1 kg, para facilitar su posterior uso en el laboratorio.

\section{Análisis de muestras en laboratorio}

Se realizaron tres tratamientos $(T)$ sobre las muestras de ambos hábitats: BS y ZP. Inicialmente, se tomaron $50 \mathrm{~g}$ de suelo de cada muestra, y se aplicaron los siguientes tratamientos, T1: con $50 \mathrm{~g}$ de fosfato tricálcico, T2: con $0.015 \mathrm{~g}$ de fosfato tricálcico, y T3: control, sin el enriquecimiento previo ${ }^{2,3,7}$. Los T fueron mantenidos durante siete días, a temperatura ambiente, después de este tiempo se procedió a la siembra de los microorganismos. De cada $\mathrm{T}$ se realizaron tres diluciones seriadas (base 10): $10^{-1}, 10^{-3}$ y $10^{-5}$, se tomaron $0.1 \mathrm{ml}$ de cada dilución, con una micropipeta, y se colocaron en cajas Petri con medio de cultivo selectivo NBRIP (Nautiyal, 1999) ${ }^{10}$, considerando tres repeticiones para cada dilución; obteniendo un total de 54 cajas Petri para crecimiento de BSF, 27 por cada hábitat. Todas las cajas sembradas se incubaron a $28^{\circ} \mathrm{C}$ durante cuatro días.

Luego del tiempo de incubación, de las 54 cajas cultivadas, se seleccionaron 20 aislados promisorios de BSF, 10 aislados por cada hábitat, para proceder con su aislamiento individual (cultivos puros) en medio NBRIP. El criterio de selección de los aislados de BSF, se basó en: 1.- presencia de halos de solubilización de $\mathrm{P}$, y 2.- diferencias morfológicas entre los aislados (color, tamaño, forma y textura).

El medio NBRIP, contiene fosfatos que al ser solubilizados por las BSF deja un halo claro alrededor de la colonia ${ }^{3}$. En los cultivos puros de los aislados promisorios de BSF se midieron el diámetro de la colonia y el diámetro del halo de solubilización, con ayuda de un calibrador, durante cuatro semanas. Estas medidas fueron utilizadas para obtener el índice de solubilización de fosfatos (ISF), mediante la fórmula propuesta por Becerra (2012) $)^{11}$ :

$$
I S F=\left(d_{c}+d_{h}\right) / d_{c}
$$

Donde, es diámetro de la colonia y es el diámetro del halo.

Todas las muestras fueron analizadas en el Centro de Investigación de Biotecnologías de Ecuador (CIBE-ESPOL), Guayaquil-Ecuador.

\section{Resultados y discusión}

Luego de la primera semana de evaluación, el desarrollo

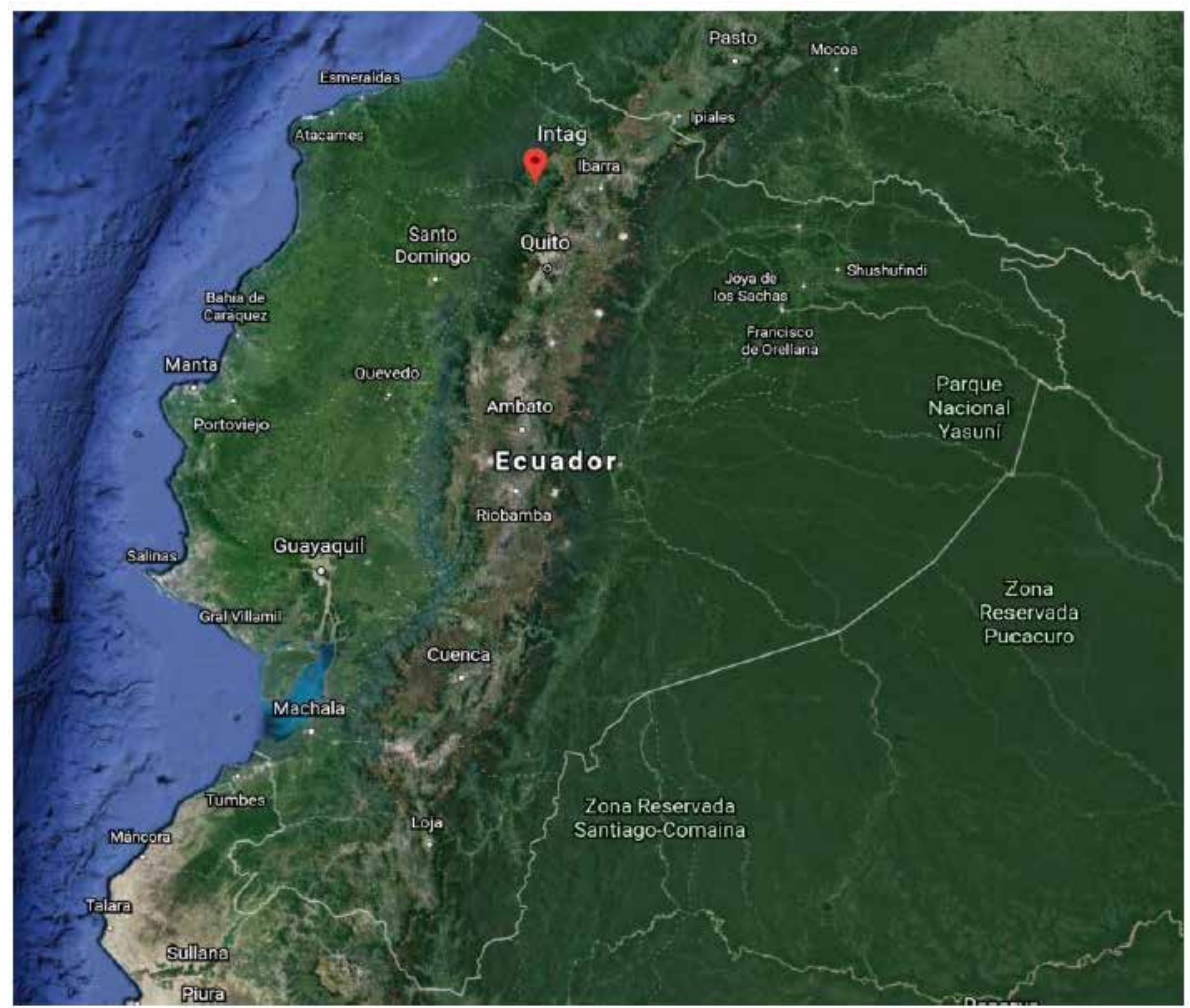

Figura 1. Área de estudio, localidad La Neblina en Intag, provincia de Imbabura (imagen Google Earth, 2020). 
del halo de solubilización del P se pudo medir en tres aislados promisorios del cultivo puro, dos provenientes de BS (BS-5 y BS-6) y uno de ZP (ZP-10) (Figura 2); mientras que el resto de aislados no mostraron un crecimiento visible del halo. Pese a la baja cantidad de aislados, se esperaba esta diferencia debido al tipo de suelo que proviene de cada hábitat ${ }^{12}$. Las muestras de suelo de la rizosfera de C. montana en BS se caracteriza por ser negro y profundo, formado por cenizas volcánicas jóvenes ${ }^{13}$, y un alto contenido de $P$, con concentraciones entre 28.27 y 29.17 ppm $^{14}$. Sin embargo, perturbaciones antrópicas podrían alterar estas características edáficas, y por ende la diversidad microbiana; según Usiche et al., la abundancia y diversidad de las BSF se ven altamente afectadas por el uso del suelo entre bosque y pastizal ${ }^{15}$.

Respecto al ISF, los mayores valores se obtuvieron en los aislados promisorios BS-5 y BS-6, con 3.43 y 2.52, respectivamente; seguido del aislado ZP-10 con un valor de 2.08 (Figura 3). Se ha demostrado que el ISF puede alcanzar valores de hasta 12.0, bajo incubación con fosfato tricálcico ${ }^{16}$. No obstante, la eficiencia de solubilización se evaluó en base a la escala formulada por Silva Filho \& Vidor ${ }^{17}$, donde los valores por encima de 3.0 se clasificaron como solubilizadores altos (BS-5), y los valores de 2.0 a 3.0 como solubilizadores medios (BS-6 y ZP-1). Aún en los aislados provenientes de BS, los ISF son distintos, posiblemente porque se trata de dos géneros bacterianos con diferente capacidad solubilizadora. Existen bacterias que utilizan diferentes mecanismos de solubilización de P, como por ejemplo la producción de ácidos orgánicos, siendo el género Bacillus sp. el más representativo de este grupo ${ }^{18}$.
Por otro lado, dado que el ISF es directamente proporcional a la actividad de solubilización de P por parte de las BSF, los aislamientos bacterianos con altos ISF resultan potencialmente efectivos para ser incluidos en el desarrollo de biofertilizantes fosfatados ${ }^{4}$; que, a partir de esta investigación, podrían ser empleados en el crecimiento ex situ de C. montana. Este podría ser el caso del aislado promisorio BS-5 que alcanzó el mayor valor de ISF, al cual se les aplicó el T1 (50 g de fosfato tricálcico), lo que sugiere una estrategia efectiva de selectividad al tratamiento empleado. Como menciona Sánchez et al. ${ }^{19}$ se puede inferir que el efecto positivo de los microorganismos se pudo deber a un aumento en la disponibilidad de $P$ en suelo, en forma de fosfato tricálcico.

Por otro lado, el aislado proveniente de suelo rizosférico de C. montana ZP no mostró crecimiento bacteriano luego de la primera semana de evaluación, y su ISF se mantuvo sin incrementos visibles (Figura 3); esto puede sugerir una degradación biológica del suelo por el exceso de perturbaciones generadas en a este tipo de hábitat ${ }^{20}$. Los pastizales son sistemas de rápido crecimiento y por lo general son deficientes en fosfatos por lo que los resultados obtenidos concuerdan con estudios donde se alteran los factores edafoclimáticos de las comunidades de bacterias solubilizadoras de fosfato inorgánico ${ }^{21,22}$.

\section{Conclusiones}

Los resultados de esta investigación confirman la presencia de BSF en la rizósfera de C. montana, en ambos hábitats,
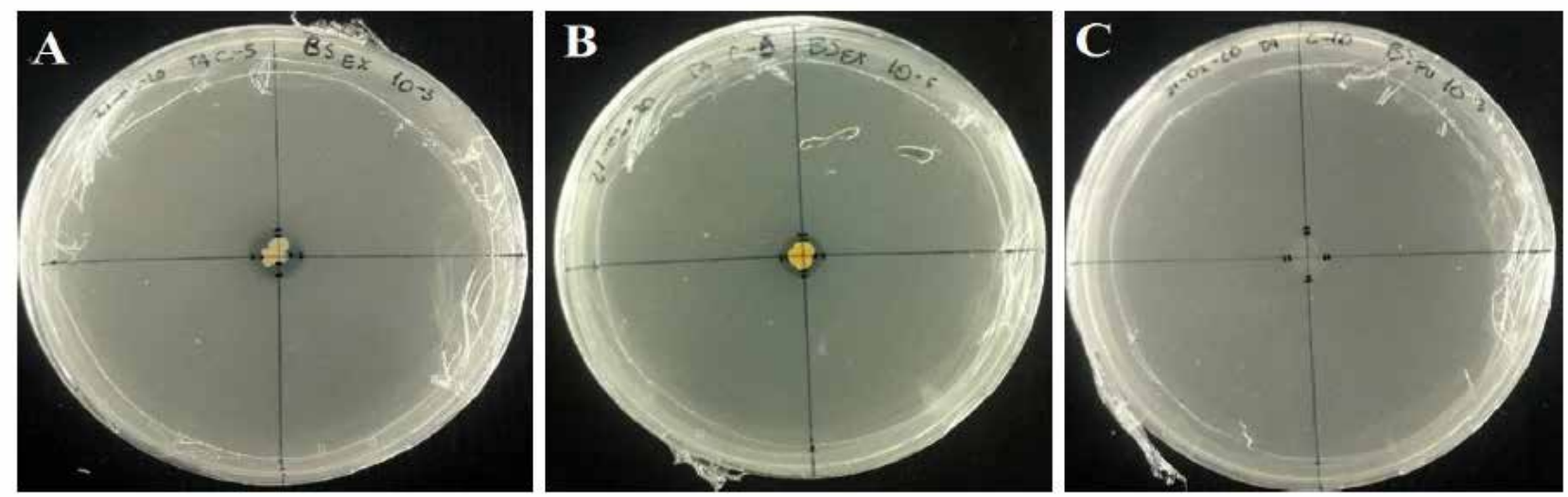

Figura 2. Aislados promisorios mostrando su halo de solubilización después de la primera semana de incubación: A) BS-5, B) BS-6 y C) ZP-10.

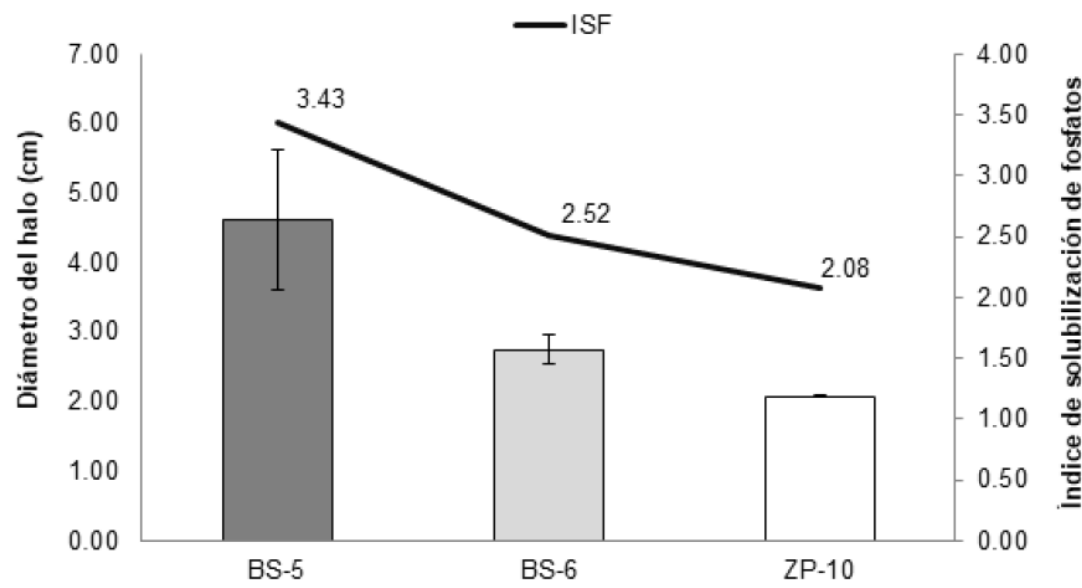

Aislados bacterianos

Figura 3. Crecimiento del halo de solubilización de los tres aislados promisorios y línea de tendencia del índice de solubilización de fosfatos (ISF), durante la cuarta semanas de evaluación. 
BS y ZP. Por otro lado, el enriquecimiento con fosfato tricálcico brinda un mejor criterio de selección para la bioprospección de microorganismos nativos en suelos rizosféricos de C. montana en BS.

Es importante mencionar que, aunque nuestro estudio muestra resultados preliminares en cuanto a la capacidad solubilizadora de BSF asociadas a dos hábitats de C. montana, con distinto grado de perturbación, nosotros podemos concluir que los aislados bacterianos promisorios obtenidos en los bosques más conservados tiene mayor capacidad solubilizadora de P inorgánico; por lo que, estos podrían servir como fuente prometedora de bioinsumos para favorecer el crecimiento de C. montana, y otras especies forestales, ex situ.

\section{Agradecimientos}

Agradecemos a Ms. C. Abigail Montero y Mg. Rodrigo Oviedo, por su contribución en las pruebas de laboratorio. La investigación fue financiada por la Corporación Ecuatoriana para el Desarrollo de la Investigación y la Academia CEPRA XIII-2019-03.

\section{Referencias bibliográficas}

1. Cater M, Parkin G, Culley J, Hao X, Ball B. Soil Density and Porosity. 2007 doi:10.1201/9781420005271.ch57.

2. Chen YP, Rekha PD, Arun AB, Shen FT, Lai WA, Young CC. Phosphate solubilizing bacteria from subtropical soil and their tricalcium phosphate solubilizing abilities. Appl Soil Ecol 2006; 34 33-41.

3. Rodríguez H, Fraga R. Phosphate solubilizing bacteria and their role in plant growth promotion. Biotechnol Adv 1999; 17: 319339.

4. Ávila Martínez EG, Lizarazo Forero LM, Cortés Pérez F. Promoción del crecimiento de baccharis macrantha (Asteraceae) con bacterias solubilizadoras de fosfatos asociadas a su rizosfera. Acta Biol Colomb 2015; 20: 121-131.

5. Volland $F$, Pucha D, Bräuning A. Hydro-climatic variability in southern Ecuador reflected by tree-ring oxygen isotopes. Erdkunde 2016; 70: 69-82.

6. CITES, UNEP. Apéndices I, II y III Convención sobre el comercio internacional de especies amenazadas de fauna y flora silvestres. 2019; 41: 80.

7. Gunter S, Weber M, Stimm B, Mosandl R. Silviculture in the Tropics. Springer Heidelberg Dordrecht London New York. 2011 http:// link.springer.com/10.1007/978-3-642-19986-8.

8. Vallejo Quintero VE. Importancia y utilidad de la evaluación de la calidad de suelos a través del componente microbiano: Experiencias en sistemas silvopastoriles. Colomb For 2013; 16: 83.

9. Valera CA, Valle Junior RF, Varandas SGP, Sanches Fernandes LF, Pacheco FAL. The role of environmental land use conflicts in soil fertility: A study on the Uberaba River basin, Brazil. Sci Total Environ 2016; 562: 463-473.

10. Nautiyal CS. An efficient microbiological growth medium for screening phosphate solubilizing microorganisms. FEMS Microbiol Lett 1999; 170: 265-270.

11. Becerra J, Quintero D, Martínez M, Matiz A. Characterization of phosphate solubilizing microorganisms isolated from soils planted with cape gooseberry (Physalis peruviana L.). Rev Colomb Ciencias Hortícolas 2011; 5: 195-208.

12. Souchie EL, Saggin OJ, Silva EMR, Campello EFC, Azcón R, Barea JM. Communities of P-solubilizing bacteria, fungi and arbuscular mycorrhizal fungi in grass pasture and secondary forest of paraty, RJ - Brazil. An Acad Bras Cienc 2006; 78: 183-193.

13. Jara Torres PP. Efectos del cambio de la cobertura vegetal y del uso de la tierra sobre la cantidad y calidad de materia orgánica del suelo en ecosistemas Alto-Andinos de Ecuador. 2018. doi:10.14201/gredos.140319

14. Cáceres H. Evaluación de las propiedades físicas, químicas y bi- ológicas del suelo en plantaciones de pinus radiata, en tres sitios de la Región Sierra Ecuatoriana. 2017.http://repositorio.utn.edu. ec/handle/123456789/7445.

15. Useche YM, Valencia H, Perez H. Caracterización de bacterias y hongos solubilizadores de fosfato bajo tres usos de suelo en el Sur del Trapecio Amazónico. Acta Biológica Colomb 2004; 9: 129-130.

16. Kandjimi OS, Uzabakiriho J, Chimwamurombe PM. Isolation and characterization of culturable bacteria from bulk soil samples and the rhizosphere of arid-adapted Tylosema esculentum (Burchell). A. Schreiber (Marama bean) in Namibia. African J Biotechnol 2015; 14: 944-952.

17. Silva Filho GN, Vidor C. Solubilização de fostatos por microrganismos na presença de fontes de carbono. Rev Bras Ciência do Solo 2000; 24: 311-319.

18. Corrales Ramírez, MSc LC, Arévalo Galvez ZY, Moreno Burbano VE. Solubilización de fosfatos: una función microbiana importante en el desarrollo vegetal. Nova 2014; 12: 67.

19. Sánchez López DB, Romero Perdomo FA, Bonilla Buitrago RR. Respuesta de Physalis peruviana L. A la inoculación con bacterias solubilizadoras de fosfato. Rev Mex Ciencias Agrícolas 2018; 5 : 901.

20.López R. Degradación del suelo: causas, procesos, evaluación e investigación. 2002.http://www.serbi.ula.ve/serbiula/libros-electronicos/Libros/degradacion/pfd/librocompleto.pdf.

21. Zeng Q, Wu X, Wang J, Ding X. Phosphate solubilization and gene expression of phosphate-solubilizing bacterium Burkholderia multivorans WS-FJ9 under different levels of soluble phosphate. J Microbiol Biotechnol 2017; 27: 844-855.

22. Zheng BX, Zhang DP, Wang Y, Hao XL, Wadaan MAM, Hozzein WN et al. Responses to soil pH gradients of inorganic phosphate solubilizing bacteria community. Sci Rep 2019; 9: 1-8.

Received: 20 noviembre 2020 Accepted: 20 enero 2021 